\title{
Effect of Touch-Screen Size on Game Immersion
}

\author{
Matt Thompson \\ Department of Computer Science \\ York \\ YO10 5GH \\ mrt507@york.ac.uk
}

\author{
A. Imran Nordin \\ Department of Computer Science \\ York \\ YO10 5GH \\ imran@cs.york.ac.uk
}

\author{
Paul Cairns \\ Department of Computer Science \\ York \\ YO10 5GH \\ paul.cairns@york.ac.uk
}

\begin{abstract}
People are now able to enjoy playing their favourite videogames on different types of devices. In this paper, we investigate the influence on players' game immersion level by changing the size of the touch screen device used. We use two different sizes of touch screen device, iPod Touch and iPad, and let people play videogames on it, measuring their immersion level. We find that the level of immersion is higher for the larger touch screen size in comparison with the smaller one. The overall picture is therefore clear and suggests that different sizes of touch screen could be an important factor to influence immersion in videogames.
\end{abstract}

game, immersion, screen size, mobile, touch screen

\section{INTRODUCTION}

Videogames are a hugely popular activity as well as a significant business sector (BBC-News 2011). The majority of the UK and US populations play videogames on a regular basis (ESA 2012). However, the question of the main factors that cause a lot of gamers to enjoy videogames is still obscure.

There are currently several approaches including immersion (Brown and Cairns 2004), Puppetry (Calvillo-Gámez et al. 2010), gaming experience (Bernhaupt et al. 2008) and GameFlow (Sweetser and Wyeth 2005) amongst others but there has been little work to synthesise these theories to bring a more unified account of the gaming experience. In addition, videogames have undergone many changes throughout their history since the first invented game in the late 1970s (Anderson and Bushman 2001). Once the pursuit of computer scientists playing text adventures on shared terminals, they have since exploded into the mainstream with the advent of consoles and arcade machines in the 1990s. These days, computer games have become more popular than ever. With the success of smartphones such as the iPhone and Android devices, a great many people are able to play games anywhere they like.

An important component of the experience of videogames is that of the feeling of being immersed in the game. As well as being commonly reported by players and reviewers, (Brown and Cairns 2004) it also features as a significant component of the other approaches to game experience. A basic understanding of what immersion is is beginning to emerge (Jennett et al. 2008) but there is still much unknown about it, in particular what aspects of gaming are important influences on the immersion of game players. Since smartphone gaming is now so popular, has this occurred despite the lack of game immersion, or because it is still attainable despite the decrease in screen size?

This paper describes an experiment investigating the relationship between the size of touch screen devices and the level of immersion whilst playing videogames. It seems that the small screen devices do reduce immersion but that this is a quite modest effect. Nonetheless, it could be an important factor when considered in relation to other factors of using these devices to play games.

\section{INFLUENCES ON IMMERSION}

The concept of immersion is difficult to define precisely. However, the grounded theory conducted by Brown and Cairns (2004) shows immersion is a phenomenon that is commonly reported among gamers, reviewers and designers of videogames. Colloquially, it is understood to be the sense of being "in the game." This is in a cognitive sense. It is possible to feel a sense of presence in virtual environments (Slater et al. 1994) where a player may feel they are in the virtual world. This however 
is distinct from immersion because some games simply do not offer a virtual world for the player to be present in and likewise players may feel present in a virtual world but not immersed in the activities they have to do there.

Immersion should also be distinguished from flow (Csikszentmihalyi 1991) especially given its supposed importance to all game experiences (Chen 2007). Flow is an optimal psychological experience which wholly absorbs the attention and efforts of a person whereas immersion is a much more prosaic experience such as might be had whilst playing a game on an iPhone whilst waiting for a bus. If players in this situation were in Flow, they would most likely miss the bus whereas most players successfully catch the bus whilst still having something of an immersive experience. Flow then can be considered to be immersion when it is at its most intense but as Brown and Cairns (2004) found, this is only a fleeting aspect of most gaming experiences.

When it comes to understanding the gaming experience, there has been substantial research in a wide range of areas about what influences the experiences that players have. For instance, people have looked at the role of aesthetic factors (Anderson and Bushman 2001), the social context (Gajadhar et al. 2008) and even narrative (Qin et al. 2009) on the gaming experience. When it comes to immersion specifically, it has been found that immersion is influenced by music (Sanders and Cairns 2010), how much gaming people do (Seah and Cairns 2008) and what players have to think about (Cox et al. 2012).

However, for those studies, the general approach has been to look at more traditional playing contexts such as on a PC or games console. The move to mobile gaming brings its own fresh questions about what influences the gaming experience. In particular, the most obvious difference between mobile and more traditional gaming is the size of the device being used and whether the limited screen size changes the experiences that players have. McCrea (2011) argues that mobiles are not only different from traditional consoles but are different from portable game consoles as well.

There has been substantial interest in the effect of screen size on the sense of presence in media experiences. Naturally this started with interest in less interactive media. Technology offered the possibility of large television displays and these were found to produce a greater sense of presence (Lombard et al. 2000). Portable devices offered a move to much smaller displays and here the effect of this is more equivocal. Bracken and Pettey (2007) found that a movie viewed on an iPod offered a greater sense of spatial presence and involvement (which corresponds to immersion in this context) than when viewed on an ordinary television. Of course, there are lots of form factors at work here, not least of which was that, at the time, iPods were relatively new technology and the findings could not discount a "Wow!"-factor. Also, televisions are very different devices from iPods and moreover the sound on the iPod was via headphones giving less opportunity for ambient distractions. Lombard et al. (2009) also looked at the differences in playing games as well as watching movies on the experiences and found that a portable device, a Sony PSP, offered less flow and arousal than a Sony Playstation 2. However, it should be noted that flow was measured by a single item question so was at best a crude measure of engagement.

Overall then, there is some indication that the gaming experience should be different comparing small screen devices with large screen devices. Whilst there is some disagreement, the general impression is that small screens offer less involving experiences than larger screens. However, in all of these studies, one of the problems has been a lack of a good, reliable measure of gaming experience. In particular, involvement has generally been measured with quite simple, unvalidated measures. In addition, the mobile experience has been mostly compared to the experience of a normal television. There are significant form factors that change between these two types of display. We have therefore decided to focus on two very similar devices, iPad and iPod Touch, so that the form factors are more tightly controlled and moreover use a well-validated measure of immersion, the Immersive Experience Questionnaire (IEQ) (Jennett et al. 2008).

\section{EXPERIMENTAL METHOD}

\subsection{Aims and Hypothesis}

The aim of this experiment is to see how the screen size of the touchscreen device a game is being played on affects the level of immersion that the player experiences. A good argument can be made for two opposite outcomes. It can be argued that a larger screen will provide a higher level of immersion due to the 'cinematic' atmosphere that it provides, or that the smaller screen will force the player to focus harder on a smaller space and so be much less aware of their surroundings. On the whole though, the literature supports the hypothesis that the larger screened device will provide a higher level of immersion. We believe that this will be the case because a larger screen lowers the barrier to game engagement, allowing the gamer to become immersed more quickly (Brown and Cairns 2004). 


\subsection{Participants}

Twenty participants were recruited, all of whom were undergraduates and postgraduates from the University of York, UK. All of them were found by opportunity sampling, picked at random from around the university campus. Of the 20 participants, 9 were women and 11 were men. Their ages ranged from 20 to 33 years of age, with a mean age of 24.93 $(S D=3.87)$. All participants had experience playing videogames before. 12 of the participants played videogames several times a week and the other 8 participants claimed that they played videogames very rarely. On average, participants spent more than an hour playing videogames for every session. Puzzle games were the most popular type of game, played by most participants.

\subsection{Design}

The experiment was a between-subjects design. The independent variable was the size of the touchscreen device that the participants were playing the game on, with two different devices (iPad and iPod Touch). The dependent variable was the level of immersion whilst playing videogames on both devices as measured by the IEQ score. Each participant was allocated randomly to one of the two groups, balanced so that ten participants were in each group.

\subsection{Materials}

The devices used were a first-generation Apple iPad and a fourth-generation Apple iPod Touch. The screen size of the iPad is $250 \mathrm{~mm}$ diagonally, with a resolution of 1024 by 768 pixels. The iPod touch has a screen size of $89 \mathrm{~mm}$ across with a resolution of 960 by 640 pixels. The games ran as native apps on both devices (rather than inside a browser). The sound on both devices came from the built-in speakers turned up to around $80 \%$ volume.

The game chosen in this study was 'Fruit Ninja' by Half Brick Studios. In this game, fruit flies across the screen and the player has to swipe their finger over it to cut it in half, as if with a sword. Bombs also occasionally appear, and the player must avoid swiping these to progress further in the game. This was a good game to choose due to the simple nature of its gameplay, requiring no prior gaming knowledge or experience. It should be just as easy for the firsttime gamers to understand and pick up as it would be for life-long gaming enthusiasts. In addition, the gameplay, controls, sound and graphics are identical for both of the devices used.

A smartphone was used to measure the time taken playing the game. The Immersive Experience Questionnaire (IEQ) consists of 31 questions related to game immersion. The questionnaire was printed out on paper and filled in by each participant after they had played the game for five minutes, along with a short demographic questionnaire covering factual matters such as age, gender, occupation and participants gaming history, including frequency of play, and average playing duration per gaming session.

Great care was taken to ensure that the environment the game was being played in was the same for every participant, with each participant playing the game in the same room with the same lighting conditions each time. The experiment was conducted during day time from $11 \mathrm{am}$ to $4 \mathrm{pm}$.

\subsection{Procedure}

The experiment was carried out in an empty meeting room usually used for video conferences. Each participant was tested individually. They were first given an informed consent form to read and sign. Then they were asked to sit in a chair with the device on the table in front of them. A brief explanation and demonstration of how to play the game followed. Then the experimenter left the room to leave the participant alone in the room with the game. For the first group, participants were asked to play the game on the iPad and for the second group the iPod Touch. Once the experimenter left the room, the timer started. After five minutes, the experimenter returned to the room and asked the participant to fill in the IEQ and demographic questionnaires.

The window blinds for the room were shut to prevent any outside disturbances from distracting participants, but participants were observed through a glass window in the room's door. Participants were also encouraged to keep the gaming device rested on the table, for both types of device.

\section{RESULTS}

All statistical analyses were performed using $R$ version 2.14.2. The immersion (IEQ) scores were calculated by adding up the numbers of the answers of each question, with questions $6,8,9,10,18$ and 20 marked negatively.

The mean immersion score (IEQ score) for participants using the iPad device was 116.6 (standard deviation 13.14, 10 participants in group), with 102.3 being the mean immersion score for the iPod touch users (standard deviation 19.3, 10 participants in group). The differences in scores are summarised in Figure 1.

Even though the questionnaire used is guaranteed to produce a normal distribution given enough samples, 
the small sample size in this case gave a substantial deviation from a normal distribution as can be seen in Figures 2 and 3.

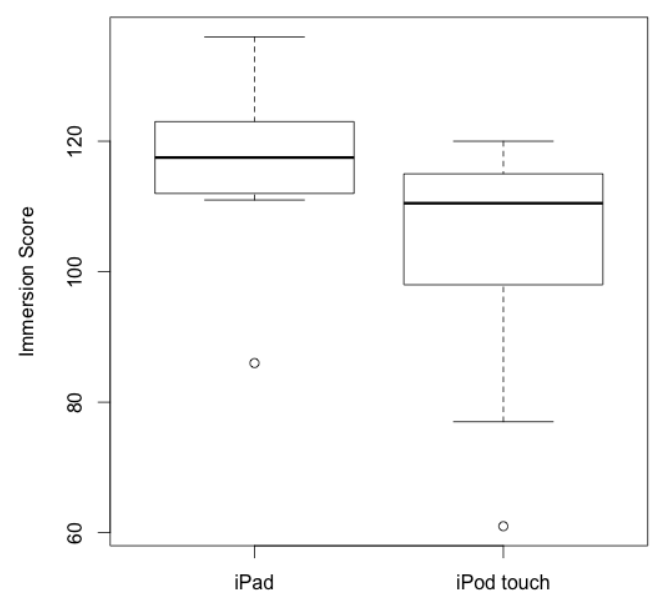

Figure 1: Box plot of immersion scores

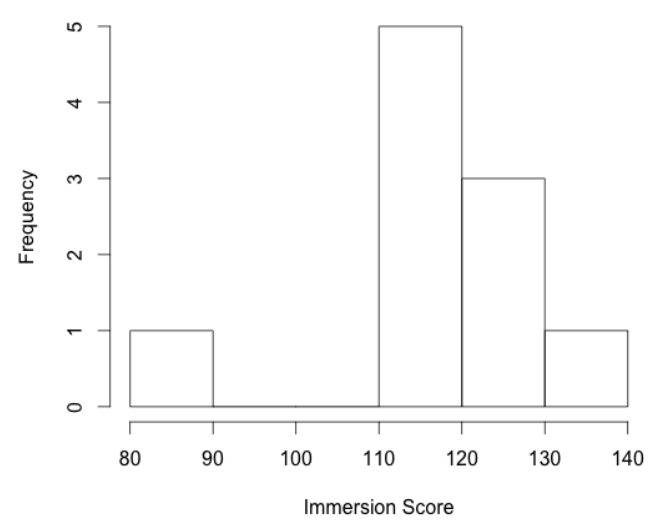

Figure 2: Histogram of iPad user immersion scores

The data was therefore analysed using a nonparametric test. Using a Mann-Whitney test, a significant $p$-value of 0.037 was obtained, with $\mathrm{W}$ $=78$. It should also be noted in Figure 1 that each group has an outlying IEQ score. There is no a priori reason to exclude these outliers and excluding them does not affect significance. They are therefore included in our analysis.

It is possible that other factors were influencing immersion along with the experimental manipulation of screen size. In particular gender differences and differences in gaming experience may be important as these can have a strong influence on the gaming experience. However, to analyse these, we must revert back to the use of parametric tests as there is

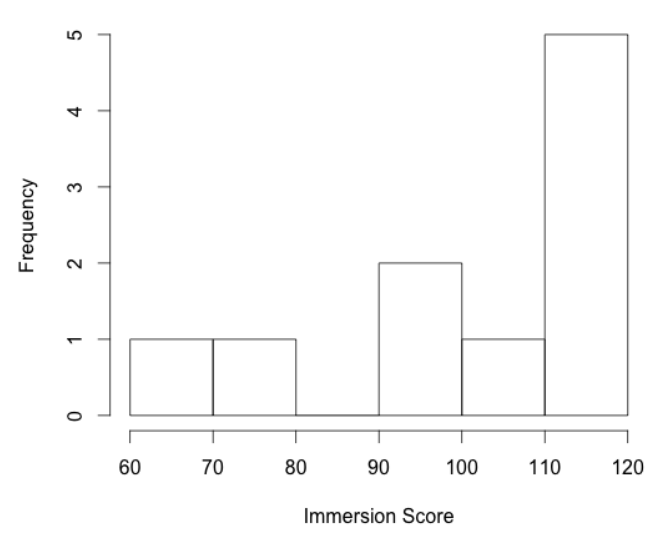

Figure 3: Histogram of iPod touch user immersion scores

no commonly accepted non-parametric test for multifactorial designs.

Testing to see if gender played a role in the results, we tried a two-way ANOVA with gender and screen size. This resulted in a $p$-value of 0.07 for the screen size, 0.72 for gender, and 0.17 for the interaction between the two. This means we can rule out gender as being an important factor in this study.

We ran another two-way ANOVA test, this time with a parameter putting subjects into two groups: those that have played the game before and were good at it, and those that had never played it or confessed they weren't skilled. In this case, the ANOVA gave $p$-values of 0.085 for screen size and 0.92 for prior experience, with the interaction between the two resulting in $p=0.68$. This too shows no statistically significant effect of prior exposure to the game in the data.

\section{DISCUSSION}

The results support the hypothesis that a larger screen influences immersion. However it should be noted that the effect is quite modest and that significance is only just achieved. Also, though these must be interpreted cautiously, the two-way analysis showed no further effect for gender or experience with the game. In these tests too, the screen size still seemed to produce some effect, irrespective of either of the other variables. This accords with the modest difference seen in the non-parametric test.

As such, this result fits with previous findings that a larger screen provides a more involving experience. This does contradict (Bracken and Pettey 2007) but here the confounds of that earlier study are much mitigated. The iPad and iPod Touch form a much more comparable device than the iPod with 
a television and also the sound was produced in the same way for each device. Additionally, these devices could now be seen as equally novel - both are well established and many of our participants would have been familiar with them, if not actually owning one or an iPhone. Thus, we feel that this study overcomes these limitations and provides a result more in accord with the experiences of using larger screens to watch television or movies.

The choice of game does seem to be a good match for the use of touch screen devices in this sort of experiment. The players had to swipe their fingers across the device screens, mimicking the slash of a sword. We believe that this interface was very unobtrusive and allowed players to become immersed in the game very quickly. The game controls certainly did not present any kind of barrier to immersion nor any particular advantage to more experienced players.

If one criticism of the game can be made, it could be that it is perhaps a very shallow, casual game. Since atmosphere and story progression are big factors in game immersion, and this game offers very little of either, perhaps it did not allow the players to become properly immersed in the game. Time was a factor in this experiment, however, and it would have been difficult to test each participant for longer than five minutes each. Given that the game was so shallow, and the time so short, perhaps not all of the players were able to enter the third stage of 'total' immersion mentioned by Brown and Cairns (2004), merely becoming engaged or engrossed with the game. Therefore, using a more story-focused game and allowing the players to play the game for longer would probably be better for testing game immersion.

A secondary consequence of this may be that for this sort of short gaming experience, the screen size has an undue influence. For longer, more involving games, it may be that screen size is irrelevant. We might also speculate that once a gamer does get immersed in a game on a smaller screen, they might be more fully immersed and less aware of their surroundings than if they were playing on a large screen because of the need to focus attention more strongly in on the small display. However, we think that it will be more difficult for them to attain this immersion because of the barrier that the smaller screen presents.

\section{CONCLUSIONS AND FURTHER WORK}

Given the small sample size in this study, we think a further investigation with more participants would be well justified. Future work could also be done to determine the role of screen resolution in game immersion. The devices used in this study had differing pixel densities, with the iPod Touch having a greater number of pixels-per-inch. This could have affected players' levels of immersion, so future studies should use devices with similar pixel densities.

On another note, we think that further studies investigating the type of game (casual versus storydriven) and how this affects immersion could be worth undertaking. It would be especially interesting to see if the screen size also has different effects on immersion levels for different types of gaming experience.

Overall then, it seems that small screens do offer a less immersive experience for touch screen devices. A much more speculative line of research would be to see if this could be mitigated by using other aspects of the game design, such as sound.

\section{ACKNOWLEDGEMENTS}

We would like to thank all the volunteers that gave up their time to participate in this experiment.

\section{REFERENCES}

Anderson, C. and Bushman, B. (2001). Effects of violent video games on aggressive behavior, aggressive cognition, aggressive affect, physiological arousal, and prosocial behavior: A meta-analytic review of the scientific literature. Psychological science, 12(5):353-359.

BBC-News (2011). Vivendi profits boosted by video games.

Bernhaupt, R., ljsselsteijn, W., Mueller, F., Tscheligi, M., and Wixon, D. (2008). Evaluating user experiences in games. In CHl'08 extended abstracts on Human factors in computing systems, pages 3905-3908. ACM.

Bracken, C. C. and Pettey, G. (2007). It is really a smaller (and smaller) world: Presence and small screens. In Presence 2007, pages 283-290. ISPR.

Brown, E. and Cairns, P. (2004). A grounded investigation of game immersion. In $\mathrm{CHl}$. 4 extended abstracts on Human factors in computing systems, pages 1297-1300. ACM.

Calvillo-Gámez, E., Cairns, P., and Cox, A. (2010). Assessing the core elements of the gaming experience. Evaluating User Experience in Games, pages $47-71$.

Chen, J. (2007). Flow in games (and everything else). Communications of the ACM, 50(4):31-34.

Cox, A., Cairns, P., Shah, P., and Carroll, M. (2012). Not doing but thinking: the role of challenge in 
the gaming experience. In Proceedings of the 2012 ACM annual conference on Human Factors in Computing Systems, pages 79-88. ACM.

Csikszentmihalyi, M. (1991). Flow: The psychology of optimal experience. Harper Perennial.

ESA (2012). Essential facts about the computer and video game industry. Retreived June, 1.

Gajadhar, B., de Kort, Y., and IJsselsteijn, W. (2008). Influence of social setting on player experience of digital games. In $\mathrm{CHI}$ '08 extended abstracts on Human factors in computing systems, CHI EA '08, pages 3099-3104, New York. ACM Press.

Jennett, C., Cox, A., Cairns, P., Dhoparee, S., Epps, A., Tijs, T., and Walton, A. (2008). Measuring and defining the experience of immersion in games. International journal of human-computer studies, 66(9):641-661.

Lombard, M., Reich, R., Grabe, M., Bracken, C. C., and Ditton, T. (2000). Presence and television: the role of screen size. Human Communication Research, 26(1):75-98.

Lombard, M., Reich, R., Grabe, M., Bracken, C. C., and Ditton, T. (2009). You cant take it with you? effects of handheld portable media consoles on physiological and psychological responses to video game and movie content. CyberPsychology and Behavior, 12(3):291-297.

McCrea, C. (2011). We play in public: The nature and context of portable gaming systems. Convergence, 17(4):389-403.

Qin, H., Rau, P.-L. P., and Salvendy, G. (2009). Measuring player immersion in the computer game narrative. Int. J. of Human-Computer Interaction, 25(2):107-133.

Sanders, T. and Cairns, P. (2010). Time perception, immersion and music in videogames. In Proceedings of the 24th BCS Interaction Specialist Group Conference, pages 160-167. British Computer Society.

Seah, M.-L. and Cairns, P. (2008). From immersion to addiction in videogames. In Proc. of $\mathrm{BCS} \mathrm{HCl}$ 2008, pages 55-63.

Slater, M., Usoh, M., and Steed, A. (1994). Depth of presence in virtual environments. PresenceTeleoperators and Virtual Environments, 3(2):130144.

Sweetser, P. and Wyeth, P. (2005). Gameflow: a model for evaluating player enjoyment in games. Computers in Entertainment (CIE), 3(3):3-3. 